\title{
Examining the Role of Psychosocial Influences on Black Maternal Health During the COVID-19 Pandemic
}

\author{
Keri Carvalho ${ }^{1}$ - Anna Kheyfets ${ }^{1} \cdot$ Blessing Lawrence $^{1} \cdot$ Alison Moky $^{1} \cdot$ Lauren Harris $^{1} \cdot$ Siwaar Abouhala $^{1}$. \\ Ndidiamaka Amutah-Onukagha ${ }^{1}$
}

Accepted: 4 June 2021 / Published online: 21 August 2021

(c) The Author(s), under exclusive licence to Springer Science+Business Media, LLC, part of Springer Nature 2021

\begin{abstract}
Introduction Due to the disproportionate impact of COVID-19 on communities of color, racial disparities in maternal mortality and morbidity are likely to increase. However, neighborhood and social support factors have yet to be discussed as potential mechanisms by which COVID-19 can exacerbate racial disparities.

Methods We examined literature on the role of neighborhood factors and social support on maternal health outcomes and provided analytical perspective on the potential impacts of COVID-19 on Black birthing people.

Results Even prior to the pandemic, Black individuals were disproportionately impacted by psychosocial stress. However, the compounding effect of pre-existing and current pandemic psychosocial stressors may be a mechanism by which racial disparities are exacerbated and result in higher rates of maternal mortality and morbidity in Black women.

Conclusion We recommend continued monitoring of data related to racial disparities in maternal mortality and morbidity throughout the pandemic. Given that Black women may be disproportionately impacted by psychosocial stress, it is necessary for leadership structures and communities to recognize the potential for worsening disparities and intervene.
\end{abstract}

Keywords Maternal racial disparities $\cdot$ COVID-19 $\cdot$ Psychosocial stress $\cdot$ Social support

\section{Signifiance}

This commentary synthesizes several ways that the COVID19 pandemic disproportionately impacted communities of color and enhanced psychosocial stressors on specifically, Black birthing people. It calls attention to the enhanced psychosocial stress that may result in exacerbations of the Black/white racial disparities in maternal outcomes and calls for careful research monitoring these trends and policyinterventions to mitigate them.

Keri Carvalho

keri.carvalho@tufts.edu

1 Department of Public Health and Community Medicine, Tufts University School of Medicine, 136 Harrison Avenue MV 252, Boston, MA 02111, USA

\section{Introduction}

The effects of racial disparities on health outcomes are more evident than ever before. COVID-19 continues to disproportionately affect communities of color. Black individuals are diagnosed with COVID-19 at higher rates and experience more severe forms of the illness (Price-Haywood et al., 2020). The age-adjusted mortality rate among Black people in the U.S. is 3.4 times greater than that of white people (Nguemeni Tiako et al., 2020). The greatest disparities are found in densely populated and historically segregated cities (Coughlin et al., 2020). Social determinants of health, such as low income and lack of access to healthcare are largely responsible for the increased rates of mortality and morbidity from COVID-19 among the Black population in the U.S. (Dorn et al., 2020). Black populations suffer higher rates of comorbidities such as obesity and diabetes, which affect the duration and severity of the illness (Gu et al., 2020). Social and community-level factors further compound disproportionate COVID-19 incidence rates within Black individuals (Selden \& Berdahl, 2020). Some of the disproportionate and community-level factors include increased housing density, 
differential ability to social distance due to occupation, and complications from comorbidities (Yancy, 2020).

Due to the disproportionate effect of COVID-19 on communities of color, an increase in racial disparities for maternal mortality and morbidity between non-Hispanic white and non-Hispanic Black individuals (hereafter referred to as white and Black respectively) in the U.S. could occur in subsequent years. Preliminary evidence of the impact of COVID-19 on pregnancy and birth outcomes show increased likelihood of cesarean deliveries and postpartum hemorrhage in patients with COVID-19 and pregnant women ${ }^{1}$ with COVID-19 are more likely to be hospitalized in the ICU than pregnant women without COVID-19 (Chi et al., 2020; Hcini et al., 2021). Prior to the pandemic, pregnancy-related mortality ratios were three to four times higher among Black women than white women (Creanga et al., 2014, 2017). Black women experienced higher rates of severe maternal morbidity than white women; 231.1 and 139.2 per 10,000 births respectively (Noursi et al., 2020). Black women also experienced a greater prevalence of preterm birth, fetal growth restriction, fetal demise, and inadequate receipt of prenatal care (Bryant et al., 2010). While studies that investigated the impact of the pandemic on maternal health outcomes found mixed results, the impact of psychosocial stress on outcomes and racial disparities during the pandemic has yet to be studied (Adhikari et al., 2020; Trocado et al., 2020). Additionally, long term outcomes of psychosocial factors are yet to be known. However, it is clear that the healthcare system is strained with overworked personnel and policies to restrict spreading the virus. This situation could compound the consequences of social determinants of health on communities of color, thereby worsening perinatal outcomes for women who have never contracted the virus (Hcini et al., 2021).

Neighborhood and social support factors have yet to be discussed as potential mechanisms by which COVID-19 can exacerbate racial disparities. As healthcare systems are overwhelmed, hospitals have changed their labor and delivery policies during the pandemic such that key support members can be excluded from the birth experience (Raman, 2020). This could leave Black women more vulnerable than they already are. This commentary will describe neighborhood, social support, and other psychosocial factors specific to Black women that may serve as mechanisms to exacerbate existing disparities in maternal mortality and morbidity during the pandemic.

\footnotetext{
1 The authors recognize that not all pregnant and birthing individuals identify as female. However, in keeping with the terminology of the most recent literature, and to accurately present findings related to women specifically, we use the term "women".
}

\section{Neighborhood Factors}

Neighborhood factors are a potential mechanism through which COVID-19 could exacerbate racial disparities in maternal health. Disparities in health outcomes based on neighborhood stem from racial segregation, limited options for exercise, and access to nutritious foods, maternity care, and reliable transportation (Taylor et al., 2019; Wolfson \& Leung, 2020).

\section{Racial Segregation}

Black women are more likely to live in segregated neighborhoods as a result of persistent residential segregation and historical redlining practices and policies (Nardone et al., 2020; Taylor et al., 2019). Consequently, communities of color commonly experience higher rates of pollution, poverty, low socioeconomic status, and increased risk of violence, which can affect the mental and physical health of pregnant and postpartum women (Giurgescu et al., 2015). Residential segregation has also been associated with high COVID-19 incidence rates (Gu et al., 2020). This may be in part due to an abundance of multigenerational homes with overcrowded living spaces unsuitable for quarantining, and increasing risk for COVID-19 (Coughlin et al., 2020; Wolfson \& Leung, 2020).

Additionally, Black residents are more likely to work in jobs deemed essential (Wolfson \& Leung, 2020). Many individuals do not have the ability to work remotely in order to practice social distancing. Even for those who are not essential workers, staying home unemployed is not an option in a low-income household (Coughlin et al., 2020). Despite country-wide indications that neighborhoods with majority Black or Hispanic populations bear the burden of COVID-19 infection, some reports show that majority of testing sites are located in wealthier, whiter neighborhoods (Coughlin et al., 2020; McMinn et al., 2020). All of these circumstances may require using public transport, which further increases risk in pregnant women for exposure to COVID-19.

\section{Maternity Care Deserts}

Issues of access to quality maternity care that permeate communities of color are especially concerning for pregnant women. Over $12 \%$ of births in the U.S. occur in maternity care deserts, defined as areas with limited or scarce access to maternity care services due to minimal availability of obstetricians and insurance coverage for maternity care (March of Dimes, 2018; Taylor et al., 2019). Maternity care deserts disproportionately impact Black and Hispanic neighborhoods, leading to low quality or no obstetric care (Hung et al., 2017; Taylor et al., 2019). 
Loss of resources in low income hospitals due to COVID19 could additionally lead to closure of obstetric wards and increase the distance women have to travel to obtain care (Taylor et al., 2019). Limitations in obstetric care can compound inequities due to transportation barriers, and potentially place pregnant women at increased risk of contracting the virus. This could also discourage the utilization of prenatal care due to transportation barriers (Wolf et al., 2020).

\section{Food Deserts}

Food security is also a significant neighborhood factor that directly impacts quality of nutrition, and is necessary for pregnant women. Food security can be defined as adequate availability and accessibility to nutritious and satiating foods (Wolfson \& Leung, 2020). This is difficult to achieve in food deserts and low-income communities. Those who face food insecurity often also work in low-paying jobs that do not provide the opportunity to work from home or take time off due to COVID-19. According to a recent survey of 1,500 individuals living under $250 \%$ of the federal poverty line in the U.S., those who reported very low food security reported being unable to take days off despite COVID-19 infection among family members (Wolfson \& Leung, 2020). Such working and living conditions could negatively impact the health and safety of low-income communities amid the COVID-19 pandemic.

Living in a food desert can increase risk for cardiovascular disease, hypertension, and obesity, placing individuals at higher risk for serious illness due to preexisting comorbidities related to COVID-19 infection (Singu et al., 2020). Likewise, mandated closure of parks and recreational spaces due to COVID-19 aggravates the existing lack of safe and affordable places to exercise in communities of color and could worsen health outcomes (Coughlin et al., 2020).

\section{Social Support}

Previous research demonstrates that practical and emotional support from interpersonal relationships (social support) is a major mediating factor in improving health in pregnancy and postpartum (Glazier et al., 2004). However, connections to loved ones and broader network connections to social services and food security are strained during the pandemic. A recent survey found that COVID-19 positive women were 11 times more likely to not have visitors during childbirth than matched COVID-19 negative controls and to report increased clinical acute stress, higher levels of pain during delivery, lower newborn weight, and an increased rate of admission of their newborns to the NICU (Mayopoulos et al., 2020). Additionally, lack of access to healthcare services persists in minority and low-income communities (Kozhimannil et al., 2016). However, research indicates that doulas can be one potential beneficial point of connection and social support for Black women during pregnancy and childbirth. Doulas can help traverse an unresponsive healthcare system that devalued Black pregnant women, including due to implicit biases amongst healthcare providers (Abbyad \& Robertson, 2011).

The drastic increase of COVID-19 cases was the impetus for banning the presence of a labor partner for women undergoing birth in several New York City hospitals (Arora et al., 2020). Women endured the psychological and physiological stress of labor and delivery alone without a partner, doula, or another support person. When a policy limited labor support in New York, midwives witnessed a surge of new requests for home births due to additional stress, uncertainty, and fear going into the delivery rooms alone (Van Syckle \& Caron, 2020). Out-of hospital births carry a higher rate of perinatal death and other adverse birth outcomes (Snowden et al., 2016). This may be particularly pronounced during the COVID-19 pandemic as Emergency Departments are stressed and Emergency Medical Services may take longer to respond if needed during a home birth. The original policy restricting labor partners was overturned, and an executive order passed to ensure that pregnant women giving birth can have one support person during childbirth (Zucker et al., 2020).

\section{Doula Support}

The limitation of having one support person can prevent Black women from choosing support of a doula during childbirth. Social support is a vital part of birth and health outcomes of women, as studies have indicated that women value and benefit from social support during labor; a labor companion, such as a doula, improves health outcomes for women by providing emotional, psychological, and practical support and advice (Bohren et al., 2017; World Health Organization, 2016). Doulas are distinctive from other members of the birth team because they provide continuous support before, during, and after pregnancy. Studies have shown that doula support is associated with higher rates of unmedicated vaginal births, lower cesarean delivery rates, and less frequent preterm births (Kozhimannil et al., 2016). Doulas commonly build rapport with pregnant individuals if services begin during pregnancy to ensure needs are met during labor. According to Kozhimannil et al. (2016), pregnant women can experience less anxiety and discomfort during labor with increased social support. Black pregnant women tend to experience more stress related to racial discrimination and higher rates of conflict with partners during pregnancy than white pregnant women (Earnshaw et al., 2013). Additionally, implicit bias among clinicians can 
affect treatment and communication with patients (FitzGerald \& Hurst, 2017).

Limiting doula inclusion on the maternal care team is specific to the hospital, as some hospitals limit patients to one visitor. This can result in the pregnant patient having to choose between a doula, partner or other forms of social support. Some hospitals recognize doulas as part of the birth team and will still allow one additional visitor with the doula. For instance, The Women's Hospital of Texas allows pregnant patients to include a doula, in addition to one named visitor, during labor after the doula successfully completes the hospital's COVID-19 screening process (The Women's Hospital of Texas, 2020). Limiting visitors during labor can lower social support during a vital point in the pregnancy journey.

During the pandemic, many women in the U.S. have faced labor and motherhood with trepidation given the lack of social support due to quarantining, social distancing, and travel bans (Abbyad \& Robertson, 2011). Pregnant individuals may feel isolated and this lack of support can be associated with postpartum depression and can compromise the health of both the mother and infant (Corrigan et al., 2015). The early postpartum period is considered to be an essential time to improve the overall health and survival of the newborn and mother; nevertheless, the World Health Organization announced that the postpartum period has received less surveillance from healthcare professionals than the prenatal and childbirth periods (Corrigan et al., 2015). These issues are further exacerbated with social distancing protocols as pregnant individuals are experiencing increased barriers in order to attain comprehensive care.

\section{Psychosocial Stress}

Taken together, it is clear that neighborhood factors and a lack of social support can be significant sources of psychosocial stress for Black women during the pandemic. Even prior to COVID-19, Black pregnant women were already disproportionately affected by psychosocial stressors, such as racial discrimination (Earnshaw et al., 2013). Pregnant women are particularly vulnerable to stress, as high-levels of stress are associated with negative health outcomes for mother and baby, such as low fetal weight and preterm birth (Lima et al., 2018). For pregnant women of color who are more likely to encounter persistent and severe stressors even prior to COVID-19, the heightened stress from neighborhood and social factors during the pandemic may result in widening disparities in maternal morbidities and mortality.

During the pandemic, rates of depressive symptoms have dramatically increased, potentially related to lack of control and unpredictability of the future, social isolation, and transitioning into new or unexpected roles (Ettman et al., 2020). One study demonstrated that pandemic stress is particularly severe for Black female young-adults (Kujawa et al., 2020). Additionally, nearly one-third of pregnant women reported high-levels of pandemic-related stress, with high-levels of stress more commonly reported by women of color (Preis et al., 2020).

There are of course common causes of pandemic-related stress across pregnant women that are not specific to race. In addition to concerns of staying healthy, pregnant and lactating individuals must begin to make decisions related to risks associated with transmitting COVID-19 through breastfeeding (Ahlers-Schmidt et al., 2020). Also, because there was full exclusion of pregnant and lactating individuals from clinical trials for the COVID-19 vaccine, many individuals may fear whether vaccination could be harmful to themselves or their babies (Ahlers-Schmidt et al., 2020; Farrell et al., 2020).

Black pregnant women may be especially vulnerable to pandemic stress due to the effect of cumulative stressors that their white counterparts may not encounter. One potential stressor may be distrust of the medical community to provide a safe and effective vaccine to Black individuals due to the historic mistreatment and continued racism present within medical settings (Warren et al., 2020). Another stressor that has been particularly exacerbated for Black women during the pandemic is financial strain. Black women have higher rates of job loss, unemployment, and approximately half the rates of recovered jobs compared to white women during the pandemic (Long et al., 2020; Schneider, 2020).

\section{Conclusion}

It is essential that researchers and health professionals carefully monitor data related to racial disparities in maternal morbidity and mortality throughout the pandemic. Black women were already disproportionately affected prior to the pandemic and the effects of pandemic-related psychosocial stress, including stress related to neighborhood and social support factors, may further worsen disparities. With an understanding of the deleterious effects of stress on maternal health outcomes and that Black pregnant women may be most vulnerable to pandemic-related stress, it is incumbent upon leadership structures and communities to intervene to reduce these disparities.

\section{Declarations}

Conflict of interest The author declare that they have no conflict of interest. 


\section{References}

Abbyad, C., \& Robertson, T. R. (2011). African American women's preparation for childbirth from the perspective of African American health-care providers. The Journal of Perinatal Education, 20(1), 45-53. https://doi.org/10.1891/1058-1243.20.1.45

Adhikari, E. H., Moreno, W., Zofkie, A. C., MacDonald, L., McIntire, D. D., Collins, R. R. J., \& Spong, C. Y. (2020). Pregnancy outcomes among women with and without severe acute respiratory syndrome coronavirus 2 infection. JAMA Network Open, 3(11), e2029256. https://doi.org/10.1001/jamanetworkopen.2020.29256

Ahlers-Schmidt, C. R., Hervey, A. M., Neil, T., Kuhlmann, S., \& Kuhlmann, Z. (2020). Concerns of women regarding pregnancy and childbirth during the COVID-19 pandemic. Patient Education and Counseling, 103(12), 2578-2582. https://doi.org/10.1016/j.pec. 2020.09.031

Arora, K. S., Mauch, J. T., \& Gibson, K. S. (2020). Labor and delivery visitor policies during the COVID-19 pandemic: Balancing risks and benefits. JAMA, 323(24), 2468. https://doi.org/10.1001/jama. 2020.7563

Bohren, M. A., Hofmeyr, G. J., Sakala, C., Fukuzawa, R. K., \& Cuthbert, A. (2017). Continuous support for women during childbirth. Cochrane Database of Systematic Reviews. https://doi.org/10. 1002/14651858.CD003766.pub6

Bryant, A. S., Worjoloh, A., Caughey, A. B., \& Washington, A. E. (2010). Racial/ethnic disparities in obstetric outcomes and care: Prevalence and determinants. American Journal of Obstetrics and Gynecology, 202(4), 335-343. https://doi.org/10.1016/j.ajog. 2009.10.864

Chi, J., Gong, W., \& Gao, Q. (2020). Clinical characteristics and outcomes of pregnant women with COVID-19 and the risk of vertical transmission: A systematic review. Archives of Gynecology and Obstetrics. https://doi.org/10.1007/s00404-020-05889-5

Corrigan, C. P., Kwasky, A. N., \& Groh, C. J. (2015). Social support, postpartum depression, and professional assistance: A survey of mothers in the midwestern United States. The Journal of Perinatal Education, 24(1), 48-60. https://doi.org/10.1891/1058-1243. 24.1.48

Coughlin, S. S., Moore, J. X., George, V., Johnson, J. A., \& Hobbs, J. (2020). COVID-19 among African Americans: From preliminary epidemiological surveillance data to public health action. American Journal of Public Health, 110(8), 1157-1159. https://doi.org/ 10.2105/AJPH.2020.305764

Creanga, A. A., Bateman, B. T., Kuklina, E. V., \& Callaghan, W. M. (2014). Racial and ethnic disparities in severe maternal morbidity: A multistate analysis, 2008-2010. American Journal of Obstetrics and Gynecology, 210(5), 435.e1-435.e8. https://doi.org/10.1016/j. ajog.2013.11.039

Creanga, A. A., Syverson, C., Seed, K., \& Callaghan, W. M. (2017). Pregnancy-related mortality in the United States, 2011-2013. Obstetrics and Gynecology, 130(2), 366-373. https://doi.org/10. 1097/AOG.0000000000002114

Earnshaw, V. A., Rosenthal, L., Lewis, J. B., Stasko, E. C., Tobin, J. N., Lewis, T. T., Reid, A. E., \& Ickovics, J. R. (2013). Maternal experiences with everyday discrimination and infant birth weight: A test of mediators and moderators among young, urban women of color. Annals of Behavioral Medicine, 45(1), 13-23. https://doi. org/10.1007/s12160-012-9404-3

Ettman, C. K., Abdalla, S. M., Cohen, G. H., Sampson, L., Vivier, P. M., \& Galea, S. (2020). Prevalence of depression symptoms in US adults before and during the COVID-19 pandemic. JAMA Network Open, 3(9), e2019686. https://doi.org/10.1001/jamanetworkopen. 2020.19686

Farrell, R., Michie, M., \& Pope, R. (2020). Pregnant women in trials of Covid-19: A critical time to consider ethical frameworks of inclusion in clinical trials. Ethics \& Human Research, 42(4), 17-23. https://doi.org/10.1002/eahr.500060

FitzGerald, C., \& Hurst, S. (2017). Implicit bias in healthcare professionals: A systematic review. BMC Medical Ethics, 18(1), 19. https://doi.org/10.1186/s12910-017-0179-8

Giurgescu, C., Misra, D. P., Sealy-Jefferson, S., Caldwell, C. H., Templin, T. N., Slaughter- Acey, J. C., \& Osypuk, T. L. (2015). The impact of neighborhood quality, perceived stress, and social support on depressive symptoms during pregnancy in African American women. Social Science \& Medicine, 130, 172-180. https://doi. org/10.1016/j.socscimed.2015.02.006

Glazier, R., Elgar, F., Goel, V., \& Holzapfel, S. (2004). Stress, social support, and emotional distress in a community sample of pregnant women. Journal of Psychosomatic Obstetrics \& Gynecology, 25(3-4), 247-255. https://doi.org/10.1080/01674820400024406

Gu, T., Mack, J. A., Salvatore, M., Prabhu Sankar, S., Valley, T. S., Singh, K., Nallamothu, B. K., Kheterpal, S., Lisabeth, L., Fritsche, L. G., \& Mukherjee, B. (2020). Characteristics associated with racial/ethnic disparities in COVID-19 outcomes in an academic health care system. JAMA Network Open, 3(10), e2025197. https://doi.org/10.1001/jamanetworkopen.2020.25197

Hcini, N., Maamri, F., Picone, O., Carod, J.-F., Lambert, V., Mathieu, M., Carles, G., \& Pomar, L. (2021). Maternal, fetal and neonatal outcomes of large series of SARS-CoV-2 positive pregnancies in peripartum period: A single-center prospective comparative study. European Journal of Obstetrics \& Gynecology and Reproductive Biology, 257, 11-18. https://doi.org/10.1016/j.ejogrb.2020.11.068

Hung, P., Henning-Smith, C. E., Casey, M. M., \& Kozhimannil, K. B. (2017). Access to obstetric services in rural counties still declining, with 9 percent losing services, 2004-14. Health Affairs (project Hope), 36(9), 1663-1671. https://doi.org/10.1377/hlthaff. 2017.0338

Kozhimannil, K. B., Vogelsang, C. A., Hardeman, R. R., \& Prasad, S. (2016). Disrupting the pathways of social determinants of health: Doula support during pregnancy and childbirth. The Journal of the American Board of Family Medicine, 29(3), 308-317. https:// doi.org/10.3122/jabfm.2016.03.150300

Kujawa, A., Green, H., Compas, B. E., Dickey, L., \& Pegg, S. (2020). Exposure to COVID-19 pandemic stress: Associations with depression and anxiety in emerging adults in the United States. Depression and Anxiety, 37(12), 1280-1288. https://doi.org/10. 1002/da.23109

Lima, S. A. M., El Dib, R. P., Rodrigues, M. R. K., Ferraz, G. A. R., Molina, A. C., Neto, C. A. P., de Lima, M. A. F., \& Rudge, M. V. C. (2018). Is the risk of low birth weight or preterm labor greater when maternal stress is experienced during pregnancy? A systematic review and meta-analysis of cohort studies. PLOS ONE, 13(7), e0200594. https://doi.org/10.1371/journal.pone.0200594

Long, H., Van Dam, A., Fowers, A., \& Shapiro, L. (2020). The COVID recession is the most unequal in modern U.S. history. The Washington Post. https://opportunityamericaonline.org/wp-content/ uploads/2020/10/THE-COVID-RECESSION-IS-THE-MOSTUNEQUAL-IN-MODERN-US-HISTORY.pdf

March of Dimes. (2018). Nowhere to go: Maternity care deserts across the U.S. https://www.marchofdimes.org/materials/Nowhere_to_ Go_Final.pdf

Mayopoulos, G., Ein-Dor, T., Li, K., Chan, S., \& Dekel, S. (2020). Giving birth under hospital visitor restrictions: Heightened acute stress in childbirth in COVID-19 positive women. In Review. https://doi.org/10.21203/rs.3.rs-112882/v1

McMinn, S., Carlsen, A., Jaspers, B., Talbot, R., \& Adeline, S. (2020). In large texas cities, access to coronavirus testing may depend on where you live. NPR. https://www.npr.org/sections/health-shots/ 2020/05/27/862215848/across-texas-black-and-hispanic-neigh borhoods-have-fewer-coronavirus-testing-sit 
Nardone, A., Chiang, J., \& Corburn, J. (2020). Historic Redlining and Urban Health Today in U.S. Cities. Environmental Justice, 13(4), 109-119. https://doi.org/10.1089/env.2020.0011

Nguemeni Tiako, M. J., Forman, H. P., \& Nunez-Smith, M. (2020). Racial health disparities, COVID-19, and a way forward for US health systems. Journal of Hospital Medicine, 16(1), 50-52.

Noursi, S., Saluja, B., \& Richey, L. (2020). Using the ecological systems theory to understand black/white disparities in maternal morbidity and mortality in the United States. Journal of Racial and Ethnic Health Disparities. https://doi.org/10.1007/ s40615-020-00825-4

Preis, H., Mahaffey, B., Heiselman, C., \& Lobel, M. (2020). Vulnerability and resilience to pandemic-related stress among U.S. women pregnant at the start of the COVID-19 pandemic. Social Science \& Medicine, 266, 113348. https://doi.org/10.1016/j.socsc imed.2020.113348

Price-Haywood, E. G., Burton, J., Fort, D., \& Seoane, L. (2020). Hospitalization and mortality among black patients and white patients with COVID-19. New England Journal of Medicine, 382(26), 2534-2543. https://doi.org/10.1056/NEJMsa2011686

Raman, S. (2020). COVID-19 amplifies racial disparities in maternal health. Roll Call. https://www.rollcall.com/2020/05/14/covid-19amplifies-racial-disparities-in-maternal-health/

Schneider, H. (2020). U.S. labor shock from pandemic hit women of color hardest; will it persist? Reuters. https://www.reuters.com/ article/us-great-reboot-data/u-s-labor-shock-from-pandemic-hitwomen-of-color-hardest-will-it-persist-idUSKBN26Q1LR

Selden, T. M., \& Berdahl, T. A. (2020). COVID-19 and racial/ethnic disparities in health risk, employment, and household composition: study examines potential explanations for racial-ethnic disparities in COVID-19 hospitalizations and mortality. Health Affairs, 39(9), 1624-1632. https://doi.org/10.1377/hlthaff.2020. 00897

Singu, S., Acharya, A., Challagundla, K., \& Byrareddy, S. N. (2020). Impact of social determinants of health on the emerging COVID19 pandemic in the United States. Frontiers in Public Health, 8 , 406. https://doi.org/10.3389/fpubh.2020.00406

Snowden, J. M., Tilden, E. L., Snyder, J., Quigley, B., Caughey, A. B., \& Cheng, Y. W. (2016). Planned out-of-hospital birth and birth outcomes. Obstetrical \& Gynecological Survey, 71(4), 203-204. https://doi.org/10.1097/01.ogx.0000481794.03595.6f

Taylor, J., Novoa, C., Hamm, K., \& Phadke, S. (2019). Eliminating racial disparities in maternal and infant mortality: A comprehensive policy blueprint. Center for American Progress. https://www. americanprogress.org/issues/women/reports/2019/05/02/469186/ eliminating-racial-disparities-maternal-infant-mortality/

The Women's Hospital of Texas. (2020). COVID-19 and pregnancy. https://womanshospital.com/covid-19/covid-19-and-pregnancy/

Trocado, V., Silvestre-Machado, J., Azevedo, L., Miranda, A., \& Nogueira-Silva, C. (2020). Pregnancy and COVID-19: A systematic review of maternal, obstetric and neonatal outcomes. The Journal of Maternal-Fetal \& Neonatal Medicine, 1-13. https:// doi.org/10.1080/14767058.2020.1781809

Van Dorn, A., Cooney, R. E., \& Sabin, M. L. (2020). COVID-19 exacerbating inequalities in the US. The Lancet, 395(10232), 12431244. https://doi.org/10.1016/S0140-6736(20)30893-X

Van Syckle, K., \& Caron, C. (2020). Women will not be forced to be alone when they are giving birth. New York Times. https://www. nytimes.com/2020/03/28/parenting/nyc-coronavirus-hospitalsvisitors-labor.html

Warren, R. C., Forrow, L., \& Hodge, D. A. (2020). Trustworthiness before trust-COVID-19 vaccine trials and the black community. New England Journal of Medicine, 383(22), e121. https://doi.org/ 10.1056/NEJMp2030033

Wolf, E. R., Donahue, E., Sabo, R. T., Nelson, B. B., \& Krist, A. H. (2020). Barriers to attendance of prenatal and well-child visits. Academic Pediatrics, S1876-2859, 30635-30635. https://doi.org/ 10.1016/j.acap.2020.11.025

Wolfson, J. A., \& Leung, C. W. (2020). Food insecurity and COVID19: disparities in early effects for US adults. Nutrients, 12(6), 1648. https://doi.org/10.3390/nu12061648

World Health Organization (WHO). (2016). Companion of choice during labour and childbirth for improved quality of care. https:// apps.who.int/iris/bitstream/handle/10665/250274/WHO-RHR-16. 10-eng.pdf;jsessionid=C416229E2871679B16D791787E1CF326

Yancy, C. W. (2020). COVID-19 and African Americans. JAMA, 323(19), 1891. https://doi.org/10.1001/jama.2020.6548

Zucker, H., Dreslin, S., \& Cuomo, A. (2020). Pregnancy and COVID19 resources for health care providers. New York State Department of Health. https://coronavirus.health.ny.gov/system/files/ documents/2020/03/covid-19pregnancyguidanceforproviders3. 21.20.pdf

Publisher's Note Springer Nature remains neutral with regard to jurisdictional claims in published maps and institutional affiliations. 